\title{
Interdisciplinary Simulation in Medical Education: A Novel Educational Technique for Managing Challenging Clinical Encounters
}

Paige Durling MD; Jihane Henni MD¹; Rachel Grimminck MD FRCPC¹; Dean Mrozowich MD; Joanna Rankin PhD2; Amber Barlow² University of Calgary Department of Psychiatry ${ }^{1}$ and Community Rehabilitation and Disability Studies ${ }^{2}$

\section{Background}

In 2018 simulations were introduced as an educational intervention to residents in psychiatry and students in Community Rehabilitation and Disability Studies (CRDS) at the University of Calgary. Ample research suggests that simulations are an effective learning tool, ${ }^{1-4}$ however there is minimal research on the depth of the learner experience and on multidisciplinary involvement in simulations. ${ }^{4}$

\section{Objective}

To evaluate the learner experience with simulation-based training.

\section{Methods}

50 CRDS students and 14 psychiatry residents who participated in simulation took part in 6 focus groups. Using a realist analysis and comparative methods, we identified relevant contexts, mechanisms and outcomes (CMO); CMO triads; and overall themes from the focus group data. The realist method looks at the contextual factors and mechanisms that may have contributed to different observed outcomes. The goal is to determine which aspects of an intervention work under which circumstances, and through which mechanisms, to provide a richer understanding of a complex intervention.

\section{Results}

The 10 most frequently identified contexts included characteristics inherent to the simulation experience (i.e. safe space, debrief and observer role); modifiable aspects (i.e. clarity of expectations); deliberately challenging aspects (i.e. challenging clinical scenarios and unfamiliar settings); and participant characteristic (i.e. past experience clinically and with simulations).

The mechanisms identified included characteristics inherent to the simulation experience (i.e. debrief, opportunity to act as an observer and apply theoretical knowledge); modifiable aspects (i.e. realism and correlation of reading to practice); and participants' subjective experiences (i.e. level of training, confidence, intimidation, feelings of empathy, sense of safety/danger, recognition of the value of

\begin{tabular}{|c|c|c|}
\hline Context & Mechanism & Outcome \\
\hline Debrief & Feelings of confidence in the scenario & Increased confidence via feedback \\
\hline $\begin{array}{l}\text { Previous experience } \\
\text { with simulations }\end{array}$ & Feelings of intimidation in the scenario & Increased confidence via feedback \\
\hline Acting as an observer & Acting as an observer & Increased confidence for future \\
\hline Acting as an observer & Acting as an observer & Increased learning \\
\hline Receiving feedback & Impact of the immediate debrief & Better retention \\
\hline $\begin{array}{l}\text { Previous experience } \\
\text { with similar scenarios }\end{array}$ & Opportunity to apply theoretical knowledge & Putting skills into practice \\
\hline Multidisciplinary feedback & Recognizing value of multi-disciplinary roles & Talking to security more \\
\hline Safe space of simulation & Sense of physical safety/danger in scenario & Creation of better safety plans \\
\hline Acting as an observer & Feelings of confidence in the scenario & Feelings of uncertainty/intimidation \\
\hline Unclear expectations & Understanding of own role/goals in simulation & Feeling disoriented \\
\hline Improved confidence & Improved learning & Uncomfortable emotions \\
\hline
\end{tabular}

multi-disciplinary roles, recognition of learning gaps, and understanding of ability to change practice).

The 10 most frequently identified outcomes were improved learning, increased confidence and self-reflection. Participants felt more able to manage future scenarios and improved their safety planning. However, feelings of uncertainty and intimidation during the simulation were noted.

Frequently identified $\mathrm{CMO}$ triads suggest that the debrief, acting as an observer and feelings of confidence/intimidation contribute to increased confidence. The debrief, acting as an observer and the opportunity to apply theoretical knowledge contribute to improved learning. Multidisciplinary feedback and encountering potentially dangerous situations resulted in real practice changes that improved safety. However, acting as an observer and unclear expectations contributed to uncomfortable emotional experiences.

\section{Conclusions}

Simulations appear to improve self-reported confidence, learning and safety planning in real clinical encounters. Enhancing the debrief and observer experience, including a multidisciplinary team, and utilizing high risk scenarios may enhance these outcomes. Uncomfortable emotional experiences are relatively common and an emphasis on clarity of expectations/role may mitigate this. Further research is needed.

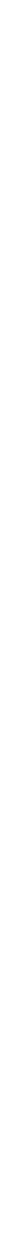

